\title{
VARIATIONS ON THE BOMAN COVERING LEMMA
}

\author{
J. M. ALDAZ
}

\begin{abstract}
We explore some variants of the Boman covering lemma, and their relationship to the boundedness properties of the maximal operator. Let $1<p<\infty$ and let $q$ be its conjugate exponent. We prove that the strong type $(q, q)$ of the uncentered maximal operator, by itself, implies certain generalizations of the Boman covering lemma for the exponent $p$, and in turn, these generalizations entail the weak type $(q, q)$ of the centered maximal operator. We show by example that it is possible for the uncentered maximal operator to be unbounded for all $1<s<\infty$, while the conclusion of the lemma holds for every $1<p<\infty$; thus, the latter condition is much weaker. Also, the boundedness of the centered maximal operator entails weak versions of the lemma.
\end{abstract}

\section{INTRODUCTION}

Often called "a useful lemma" or a "rather well-known lemma", the Boman covering lemma appears for the first time in [Bom, but [Boj] seems to be the standard reference (I have not have access to [Bom, a technical report). Hence, I will refer to [Boj] in what follows.

Closely connected to what nowadays is called the Boman chain condition, the lemma has been used to extend inequalities of Sobolev and Poincaré type to a wide class of domains (both in euclidean spaces and in several kinds of metric measure spaces) to explore the fine properties of functions belonging to different function spaces, and even to seemingly unrelated areas, such as complex dynamics. Among the many papers that could be cited here, we mention [iwNo], [Ch], Ch1], [ChWh], [hVa], HeKo], KoShSt].

In view of its usefulness it seems advisable, first, to name the lemma after its author, and second, to explore possible variations and generalizations, studying their relationships with the boundedness properties of maximal operators, both centered and uncentered.

Given $1<p<\infty$ and Lebesgue measure on $\mathbb{R}^{d}$, the Boman covering Lemma tells us that the overlap of cubes (with sides parallel to the coordinate axes) when we increase their radii, grows in a controlled manner in the $L^{p}$ norm (see Lemma 3.1 below for the precise statement). The result is an immediate consequence of Hölder's inequality and the boundedness of the uncentered maximal operator. Of course, the same proof works for metric measure spaces with a doubling measure.

Here we study alternative formulations of the lemma where doubling is not assumed. After some fairly standard definitions in Section 2, Section 3 considers variants of Boman's lemma.

2000 Mathematical Subject Classification. 42B25.

The author was partially supported by Grant MTM2015-65792-P of the MINECO of Spain, and also by by ICMAT Severo Ochoa project SEV-2015-0554 (MINECO). 
A normalized version is presented in Theorem 3.2, and in Theorem 3.6, a generalization where the smaller sets need not be balls, but can be any measurable sets with positive measure. In both cases, the usual proof works without the doubling hypothesis, and the result is obtained solely from the boundedness of the uncentered maximal operator $M^{u}$. This is interesting, since there are known boundedness results about $M^{u}$ which do not require doubling, often using instead strong uniformity assumptions on the measure $\mu$ (see [I] ) or weaker uniform assumptions on $\mu$ but strong assumptions on the space (cf. [Al1]). Also, assuming only the boundedness of the centered maximal operator $M$, it is possible to prove a weak version of the lemma, valid only for some sufficiently large expansions, which depend on the specific sequence of balls being considered, cf. Theorem 3.12.

Let $p$ and $q$ be dual exponents. Since from the conclusion of Theorem 3.6 for the exponent $q$, one can deduce the weak type $(p, p)$ of the centered maximal operator $M$ (cf. Theorem 4.5) it is natural to ask what are the relative strenghts of these properties. We prove that the conclusion of Theorem 3.2 is strictly weaker than the strong type $(p, p)$ of $M^{u}$, cf. Theorem 4.12. In the example considered there, $M^{u}$ is unbounded on $L^{s}$ for all $1 \leq s<\infty$, while the conclusion of Theorem 3.2 holds for all $1 \leq q<\infty$.

Thus, in principle it should be possible, in cases where the boundedness of $M^{u}$ either fails or is not known, to obtain boundedness results for the centered maximal function by directly proving the conclusions of Theorems 3.2 or 3.6.

I am indebeted to an anonymous referee for pointing out relevant bibliography.

\section{Definitions And notation}

In this section we introduce the basic definitions. Additional ones will be presented when needed. We will use $B(x, r):=\{y \in X: d(x, y)<r\}$ to denote open balls, $\overline{B(x, r)}$ to denote their topological closures, and $B^{c l}(x, r):=\{y \in X: d(x, y) \leq r\}$ to refer to metrically closed balls (consider $\overline{B(0,1)}$ in $\mathbb{Z}$ to see the difference). It is always assumed that measures are not identically 0 , and radii $r$ are always taken to be $>0$.

Sometimes results are valid both for collections of open balls, for collections of closed balls, and for mixed collections. To indicate this, we use the notation $U(x, r)$ for balls when we do not want to specify whether they are open or closed. Thus, $U(x, r)$ stands either for $B(x, r)$ or for $B^{c l}(x, r)$. However, when dealing with contractions or expansions, we take $U(x, r)$ and $U(x, t r), t>0$, to be both open or both closed. We do this since sometimes we allow $t=1$ in the statements of results (but the convention could be relaxed if it is assumed that $t \neq 1$ ).

Definition 2.1. A Borel measure is $\tau$-additive or $\tau$-smooth, if for every collection $\left\{U_{\alpha}: \alpha \in\right.$ $\Lambda$ \} of open sets,

$$
\mu\left(\cup_{\alpha} U_{\alpha}\right)=\sup _{\mathcal{F}} \mu\left(\cup_{i=1}^{n} U_{\alpha_{i}}\right),
$$

where the supremum is taken over all finite subcollections $\mathcal{F}=\left\{U_{\alpha_{1}}, \ldots, U_{\alpha_{n}}\right\}$ of $\left\{U_{\alpha}: \alpha \in\right.$ $\Lambda\}$. We say that $(X, d, \mu)$ is a metric measure space if $\mu$ is a $\tau$-additive Borel measure on the metric space $(X, d)$, such that $\mu$ assigns finite measure to bounded Borel sets. 
From now on we always assume that measures are locally finite (finite on bounded sets). For motivation regarding the definition of metric measure spaces using $\tau$-additivity, cf. [Al3]. Note that in separable metric spaces all Borel measures are $\tau$-additive, and the same happens with all Radon measures in arbitrary metric spaces.

Definition 2.2. Let $(X, d, \mu)$ be a metric measure space and let $g$ be a locally integrable function on $X$. For each $x \in X$, the centered Hardy-Littlewood maximal operator $M_{\mu}$ is given by

$$
M_{\mu} g(x):=\sup _{\left\{r>0: 0<\mu\left(B^{c l}(x, r)\right)\right\}} \frac{1}{\mu\left(B^{c l}(x, r)\right)} \int_{B^{c l}(x, r)}|g| d \mu .
$$

The uncentered Hardy-Littlewood maximal operator $M_{\mu}^{u}$ is defined via

$$
M_{\mu}^{u} g(x):=\sup _{\left\{r>0, y \in X: d(x, y)<r \text { and } \mu B(y, r)^{c l}>0\right\}} \frac{1}{\mu\left(B(y, r)^{c l}\right)} \int_{B(y, r)^{c l}}|g(u)| d \mu(u) .
$$

When the supremum is taken over radii $r>R$, for some fixed $R>0$, we obtain the following localized maximal operators:

$$
M_{\{R<r\}, \mu} g(x):=\sup _{\left\{r>R: 0<\mu\left(B^{c l}(x, r)\right)\right\}} \frac{1}{\mu\left(B^{c l}(x, r)\right)} \int_{B^{c l}(x, r)}|g| d \mu .
$$

In the uncentered case, the definition of $M_{\{R<r\}, \mu}^{u}$ is entirely analogous.

Sometimes it is convenient to use open balls in the definition, instead of closed balls. This does not change the values of the Hardy-Littlewood maximal operators. Hence, we could also use $U(x, r)$ and take the supremum over both open and closed balls. The reason why we define their localized versions using $r>R$ instead of $r \geq R$, is so that the values of $M_{\{R<r\}, \mu}$ and $M_{\{R<r\}, \mu}^{u}$ do not change either, when we use open or closed balls.

If we consider only one measure $\mu$, we often omit it from the notation, writing, for instance, $M$ instead of $M_{\mu}$.

Definition 2.3. A Borel measure $\mu$ on $(X, d)$ is doubling if there exists a $C \geq 1$ such that for all $r>0$ and all $x \in X, \mu(B(x, 2 r)) \leq C \mu(B(x, r))<\infty$.

Definition 2.4. Let $V, W$ be vector spaces over $\mathbb{R}$ or $\mathbb{C}$, let $C \subset V$ be a cone and let $C^{\prime} \subset W$ be an ordered cone. We say that $T: C \rightarrow C^{\prime}$ is sublinear if for all $u, v \in C$ and all $t>0$, $T(t u+v) \leq t T(u)+T(v)$.

Definition 2.5. Given $p$ with $1 \leq p<\infty$, a sublinear operator $T$ satisfies a weak type $(p, p)$ inequality if there exists a constant $c>0$ such that

$$
\mu(\{T g>\alpha\}) \leq\left(\frac{c\|g\|_{L^{p}(\mu)}}{\alpha}\right)^{p}
$$

where $c=c(p, \mu)$ depends neither on $g \in L^{p}(\mu)$ nor on $\alpha>0$. The lowest constant $c$ that satisfies the preceding inequality is denoted by $\|T\|_{L^{p} \rightarrow L^{p, \infty}}$. Likewise, if there exists a 
constant $c>0$ such that

$$
\|T g\|_{L^{p}(\mu)} \leq c\|g\|_{L^{p}(\mu)},
$$

we say that $T$ satisfies a strong type $(p, p)$ inequality. The lowest such constant (the operator norm) is denoted by $\|T\|_{L^{p} \rightarrow L^{p}}$.

\section{VARIATIONS ON THE BOMAN COVERING LEMMA}

Given $1<p<\infty$, denote by $q=p /(p-1)$ its conjugate exponent. Next we state the Boman covering lemma as presented in [Boj, Lemma 4.2].

Lemma 3.1. (Boman) Let $F=\left\{Q_{\alpha}\right\}$ be an arbitrary family of cubes in $\mathbb{R}^{d}$ (indexed by some parameter $\alpha \in I)$. Assume that for each $Q_{\alpha}$ we are given a non-negative number $a_{\alpha}$. Then for $1<p<\infty$ and $B \geq 1$ we have

$$
\left\|\sum_{\alpha} a_{\alpha} \mathbf{1}_{B Q_{\alpha}}\right\|_{L^{p}} \leq D\left\|\sum_{\alpha} a_{\alpha} \mathbf{1}_{Q_{\alpha}}\right\|_{L^{p}}
$$

where the constant $D$ depends on $d, p$ and $B$ only.

In this section we explore different versions of Lemma 3.1. The next result is a restatement of the lemma in a "normalized" manner, so while the proof is the same (an exercise given two hints, according to [HeKo, p. 67]) the doubling hypothesis is avoided, and the result follows directly from the bounds for the uncentered maximal operator (we mention that under stronger doubling hypotheses, a version of Boman's covering lemma for open sets in $\mathbb{R}^{d}$ appears in $[\mathrm{Bu}$, Lemma 2.3]).

Unlike Lemma 3.1, where the expansion factor $B$ is the same for every ball, the following variant allows different expansion factors $t_{n}$ for different balls. While the next result is stated for finite or countable collections of balls only, rather than arbitrary collections, we point out that if the collection of balls (all of them of positive measure) is uncountable, and $a_{\alpha}>0$ for an uncountable set of indices, then the right hand side of (10) is always infinite, and the inequality is trivially satisfied (more details in remark 3.3 below) so there is no real loss of generality in considering only countable (or even finite) collections of balls.

Recall that $U(x, r)$ denotes a ball of center $x$ and radius $r$, without specifying whether it is open or closed (but contractions or expansions of balls are assumed to have the same character as the original ones).

Theorem 3.2. Let $(X, d, \mu)$ be a metric measure space, fix $1<p<\infty$, and set $q=p /(p-1)$. Let $\left\{w_{n}\right\}_{n \in I}$ be a finite or countably infinite sequence of weights, so $w_{n}>0$, let $\left\{t_{n}\right\}_{n \in I}$ be a corresponding finite or countably infinite sequence of dilations, so $t_{n} \geq 1$, and let $\left\{U\left(x_{n}, r_{n}\right)\right\}_{n \in I}$ be a sequence of balls with $\mu U\left(x_{n}, r_{n}\right)>0$ for $1 \leq n<N$. Then

$$
\left\|\sum_{n \in I} \frac{w_{n} \mathbf{1}_{U\left(x_{n}, t_{n} r_{n}\right)}}{\mu U\left(x_{n}, t_{n} r_{n}\right)}\right\|_{L^{p}(\mu)} \leq\left\|M_{\mu}^{u}\right\|_{L^{q}(\mu) \rightarrow L^{q}(\mu)}\left\|\sum_{n \in I} \frac{w_{n} \mathbf{1}_{U\left(x_{n}, r_{n}\right)}}{\mu U\left(x_{n}, r_{n}\right)}\right\|_{L^{p}(\mu)} .
$$


Proof. Of course, if $\left\|M_{\mu}^{u}\right\|_{L^{q}(\mu) \rightarrow L^{q}(\mu)}=\infty$, there is nothing to show, so assume otherwise. Recalling that $\|f\|_{p}=\sup _{\|g\|_{q}=1} \int f g$, we fix $h \geq 0$ with $\|h\|_{q}=1$. Then

$$
\begin{aligned}
& \int\left(\sum_{n \in I} \frac{w_{n} \mathbf{1}_{U\left(x_{n}, t_{n} r_{n}\right)}}{\mu U\left(x_{n}, t_{n} r_{n}\right)}\right) h d \mu=\sum_{n \in I} \frac{w_{n}}{\mu U\left(x_{n}, t_{n} r_{n}\right)} \int h \mathbf{1}_{U\left(x_{n}, t_{n} r_{n}\right)} d \mu \\
& \leq \sum_{n \in I} w_{n} \inf _{x \in U\left(x_{n}, r_{n}\right)} M^{u} h(x) \leq \sum_{n \in I} \frac{w_{n}}{\mu U\left(x_{n}, r_{n}\right)} \int M^{u} h \mathbf{1}_{U\left(x_{n}, r_{n}\right)} d \mu \\
& =\int\left(\sum_{n \in I} \frac{w_{n} \mathbf{1}_{U\left(x_{n}, r_{n}\right)}}{\mu U\left(x_{n}, r_{n}\right)}\right) M^{u} h d \mu \leq\left\|\sum_{n \in I} \frac{w_{n} \mathbf{1}_{U\left(x_{n}, r_{n}\right)}}{\mu U\left(x_{n}, r_{n}\right)}\right\|\left\|_{p}\right\| M_{\mu}^{u} h \|_{q} .
\end{aligned}
$$

Taking the supremum over all $h \geq 0$ with $\|h\|_{q}=1$, yields the result.

Remark 3.3. Let $(X, d, \mu)$ be a metric measure space and let $x \in X$. We use repeatedly the fact that countable unions of countable collections are countable, in order to show that if $w_{\alpha}>0$ for uncountably many indices $\alpha$, then

$$
\left\|\sum_{\alpha \in I} \frac{w_{\alpha} \mathbf{1}_{U\left(x_{\alpha}, r_{\alpha}\right)}}{\mu U\left(x_{\alpha}, r_{\alpha}\right)}\right\|_{p}=\infty .
$$

Note first that there is a $T>0$ such that for an uncountable set $I_{1}$ of $\alpha^{\prime}$ 's, $r_{\alpha}<T, w_{\alpha}>1 / T$, and $U\left(x_{\alpha}, r_{\alpha}\right) \subset U(x, T)$. It now follows from Jensen's inequality that

$$
\begin{gathered}
\frac{1}{\mu(U(x, T))^{1 / p}}\left\|\sum_{\alpha \in I} \frac{w_{\alpha} \mathbf{1}_{U\left(x_{\alpha}, r_{\alpha}\right)}}{\mu U\left(x_{\alpha}, r_{\alpha}\right)}\right\|_{p} \geq\left\|\frac{\mathbf{1}_{U(x, T)}}{\mu(U(x, T))^{1 / p}} \sum_{\alpha \in I_{1}} \frac{w_{\alpha} \mathbf{1}_{U\left(x_{\alpha}, r_{\alpha}\right)}}{\mu U\left(x_{\alpha}, r_{\alpha}\right)}\right\|_{p} \\
\geq\left\|\frac{\mathbf{1}_{U(x, T)}}{\mu(U(x, T))} \sum_{\alpha \in I_{1}} \frac{w_{\alpha} \mathbf{1}_{U\left(x_{\alpha}, r_{\alpha}\right)}}{\mu U\left(x_{\alpha}, r_{\alpha}\right)}\right\|_{1}=\sum_{\alpha \in I_{1}} w_{\alpha}=\infty .
\end{gathered}
$$

Remark 3.4. Of course, by monotone convergence one can pass from finite to countable collections of balls, weights and dilations, so from now on we only consider finite sequences in the statements of results.

Remark 3.5. As indicated in the introduction, there are several cases where the boundedness of the uncentered maximal operator is known to hold without the measure being doubling, often using instead strong uniformity assumptions. For instance, it is shown in II , Theorem 1a] that for non-compact symmetric spaces, $M^{u}$ is bounded from $L^{q}$ to $L^{q}$ in the sharp range of exponents $q \in(2, \infty]$ (not surprisingly, the centered operator $M$ has better boundedness properties, cf. [Str]). On the other hand, local comparability (a rather mild uniformity condition) of the measure on a geometrical doubling metric space does imply the weak type $(1,1)$ of $M^{u}$ (cf. [Al1, Theorem 5.1], and [Al2] for additional information on local comparability). 
Next we present a more general version of Theorem [3.2, by examining the proof and assuming precisely what is needed. In particular, only the uncentered maximal operator localized at large radii is required to be bounded, and the smaller sets (not necessarily balls) only need to be contained in the corresponding (larger) balls (and have positive measure).

Theorem 3.6. Let $(X, d, \mu)$ be a metric measure space, fix $1<p<\infty$, and set $q=p /(p-1)$. Let $R>0$, let $\left\{w_{n}\right\}_{1 \leq n \leq N}$ be a finite sequence of weights, let $\left\{E_{n}\right\}_{1 \leq n \leq N}$ be a sequence of sets of positive measure, and let $\left\{U\left(x_{n}, r_{n}\right)\right\}_{1 \leq n \leq N}$ be a sequence of balls such that for $1 \leq n \leq N$, $r_{n}>R$ and $E_{n} \subset U\left(x_{n}, r_{n}\right)$. Then

$$
\left\|\sum_{1 \leq n \leq N} \frac{w_{n} \mathbf{1}_{U\left(x_{n}, r_{n}\right)}}{\mu U\left(x_{n}, r_{n}\right)}\right\|_{L^{p}(\mu)} \leq\left\|M_{\{R<r\}, \mu}^{u}\right\|_{L^{q}(\mu) \rightarrow L^{q}(\mu)}\left\|\sum_{1 \leq n \leq N} \frac{w_{n} \mathbf{1}_{E_{n}}}{\mu E_{n}}\right\|_{L^{p}(\mu)} .
$$

Proof. Arguing as before, we note that for $1 \leq n \leq N$, given $h \geq 0$ with $\|h\|_{q}=1$, we have

$$
\begin{gathered}
\frac{w_{n}}{\mu U\left(x_{n}, r_{n}\right)} \int h \mathbf{1}_{U\left(x_{n}, r_{n}\right)} d \mu \leq w_{n} \inf _{x \in U\left(x_{n}, r_{n}\right)} M_{\{R<r\}, \mu}^{u} h(x) \\
\leq w_{n} \inf _{x \in E_{n}} M_{\{R<r\}, \mu}^{u} h(x) \leq \frac{w_{n}}{\mu E_{n}} \int M_{\{R<r\}, \mu}^{u} h \mathbf{1}_{E_{n}} d \mu .
\end{gathered}
$$

Remark 3.7. Note that if we replace $\left\|M_{\{R<r\}, \mu}^{u}\right\|_{L^{q}(\mu) \rightarrow L^{q}(\mu)}$ in (10) by $\left\|M_{\mu}^{u}\right\|_{L^{q}(\mu) \rightarrow L^{q}(\mu)}$, then the reference to $R>0$ in the preceding theorem can be eliminated.

Under stronger hypotheses, and up to some constants, it is possible to reverse the inequality in Boman's covering lemma. Note that the doubling condition entails not only that the usual maximal functions are bounded, but boundedness also holds for the larger operators obtained by integrating over expanded balls.

Definition 3.8. Let $(X, d, \mu)$ be a metric measure space and let $g$ be a locally integrable function on $X$. Given $t \geq 1$ we set, for each $x \in X$,

$$
M_{\mu}^{t} g(x):=\sup _{\left\{r>0: 0<\mu\left(B^{c l}(x, r)\right)\right\}} \frac{1}{\mu\left(B^{c l}(x, r)\right)} \int_{B^{c l}(x, t r)}|g| d \mu .
$$

The corresponding uncentered version is defined via

$$
M_{\mu}^{u t} g(x):=\sup _{\left\{r>0, y \in X: d(x, y)<t r \text { and } \mu B^{c l}(y, r)>0\right\}} \frac{1}{\mu\left(B^{c l}(y, r)\right)} \int_{B^{c l}(y, t r)}|g(u)| d \mu(u) .
$$

It is well known that if $\mu$ is doubling with constant $C$, then $M^{u}$ is of weak type $(1,1)$ with constant $C^{3}$ (by a $5 B$-covering argument, where the radii of balls is expanded by 5 ). Likewise, $M$ is of weak type $(1,1)$ with constant $C^{2}$, by a $3 B$-covering argument. By interpolation, it follows that if $1<p<\infty$, then

$$
\left\|M_{\mu}^{u}\right\|_{L^{p} \rightarrow L^{p}} \leq \frac{C^{3 / p} p}{p-1} \quad \text { and } \quad\left\|M_{\mu}\right\|_{L^{p} \rightarrow L^{p}} \leq \frac{C^{2 / p} p}{p-1}
$$


(cf. Gra, p. 46, Exercise 1.3.3 (a)]).

Lemma 3.9. Let $(X, d, \mu)$ be a metric measure space, where $\mu$ is doubling with constant $C$. Fix $t>1$ and let $k \geq 0$ be the unique integer that satisfies $2^{k-1}<t \leq 2^{k}$. Then $M_{\mu}^{u t}$ is of weak type $(1,1)$ with constant $C^{3+k}$, and $M_{\mu}^{t}$ is of weak type $(1,1)$ with constant $C^{2+k}$. Hence $\left\|M_{\mu}^{u t}\right\|_{L^{p} \rightarrow L^{p}} \leq \frac{p}{p-1} C^{(3+k) / p}$, and $\left\|M_{\mu}^{t}\right\|_{L^{p} \rightarrow L^{p}} \leq \frac{p}{p-1} C^{(2+k) / p}$.

Proof. This is immediate from the pointwise inequalities $M_{\mu}^{u t} g(x) \leq C^{k} M_{\mu}^{u} g(x)$ and $M_{\mu}^{t} g(x) \leq$ $C^{k} M_{\mu}^{u} g(x)$, for every locally integrable $g$.

Since for any $t \geq 1$,

$$
\frac{1}{\mu U(x, r)} \int h \mathbf{1}_{U(x, r)} d \mu \leq \inf _{x \in U(x, t r)} M_{\mu}^{u t} h(x)
$$

with the same proof as before, we get the following

Theorem 3.10. (Reverse Boman) Let $(X, d, \mu)$ be a metric measure space, with $\mu$ doubling, fix $1<p<\infty$, and set $q=p /(p-1)$. Let $\left\{w_{n}\right\}_{1 \leq n \leq N}$ be a finite sequence of weights, let $\left\{t_{n}\right\}_{1 \leq n \leq N}$ be a sequence of dilations, with $1 \leq t_{n} \leq t$, and let $\left\{U\left(x_{n}, r_{n}\right)\right\}_{1 \leq n \leq N}$ be a sequence of balls. Then

$$
\left\|\sum_{1 \leq n \leq N} \frac{w_{n} \mathbf{1}_{U\left(x_{n}, r_{n}\right)}}{\mu U\left(x_{n}, r_{n}\right)}\right\|_{p} \leq\left\|M_{\mu}^{u t}\right\|_{L^{q}(\mu) \rightarrow L^{q}(\mu)}\left\|\sum_{1 \leq n \leq N} \frac{w_{n} \mathbf{1}_{U\left(x_{n}, t_{n} r_{n}\right)}}{\mu U\left(x_{n}, t_{n} r_{n}\right)}\right\|_{p} .
$$

Example 3.11. Note that the bounds given in (15) must necessarily grow with $t$, as can be seen even from the simplest example: Consider Lebesgue measure $\lambda$ on $\mathbb{R}$; let $w_{i}=1$, and $t_{i}=t=2^{k}$. Then

$$
\left\|\frac{\mathbf{1}_{B(0,1)}}{\lambda B(0,1)}\right\|_{p}=2^{-1 / q}=2^{k / q}\left\|\frac{\mathbf{1}_{B\left(0,2^{k}\right)}}{\lambda B\left(0,2^{k}\right)}\right\|_{p} .
$$

Recall that both the centered and the uncentered maximal functions are lower semicontinuous; in the case of the uncentered maximal function this is obvious; in the centered case it follows from the fact that if $c<\frac{1}{\mu B^{c l}(x, r)} \int_{B^{c l}(x, r)}|f| d \mu$, then there exists a $t>1$ such that $c<\frac{1}{\mu B^{c l}(x, t r)} \int_{B^{c l}(x, r)}|f| d \mu$.

Next we relax the boundedness hypothesis on the uncentered operator $M^{u}$ to the boundedness of the centered operator $M$. In exchange, the result is no longer obtained for arbitrary contractions $t<1$ on the right hand side: they must be "small enough".

Theorem 3.12. Let $(X, d, \mu)$ be a metric measure space, fix $1<p<\infty$, and set $q=p /(p-1)$. Let $\left\{B^{\text {cl }}\left(x_{n}, r_{n}\right)\right\}_{1 \leq n \leq N}$ be a sequence of closed balls with centers in the support of $\mu$. If $\left\|M_{\mu}\right\|_{L^{q}(\mu) \rightarrow L^{q}(\mu)}<\infty$, then there exists a numerical sequence $\left\{T_{n}\right\}_{1 \leq n \leq N}$, with $0<T_{n}<1$ depending on $\left\{B^{c l}\left(x_{n}, r_{n}\right)\right\}_{1 \leq n \leq N}$, such that whenever $0<t_{n} \leq T_{n}$, for every sequence of weights $\left\{w_{n}\right\}_{1 \leq n \leq N}$, we have 


$$
\left\|\sum_{1 \leq n \leq N} \frac{w_{n} \mathbf{1}_{B^{c l}\left(x_{n}, r_{n}\right)}}{\mu B^{c l}\left(x_{n}, r_{n}\right)}\right\|_{p} \leq 2\left\|M_{\mu}\right\|_{L^{q}(\mu) \rightarrow L^{q}(\mu)}\left\|\sum_{1 \leq n \leq N} \frac{w_{n} \mathbf{1}_{B^{c l}\left(x_{n}, t_{n} r_{n}\right)}}{\mu B^{c l}\left(x_{n}, t_{n} r_{n}\right)}\right\|_{p} .
$$

Proof. Fix $h \geq 0$ with $\|h\|_{q}=1$, select $0<\delta_{n}<r_{n}$ so that $\mu B^{c l}\left(x_{n}, \delta_{n}+r_{n}\right)<2 \mu B^{c l}\left(x_{n}, r_{n}\right)$ (here we use the fact that balls are assumed to be closed) and set $T_{n}:=\delta_{n} / r_{n}$. Then, for all $0<t_{n} \leq T_{n}$, and all $y \in B^{c l}\left(x_{n}, t_{n} r_{n}\right) \subset B^{c l}\left(x_{n}, \delta_{n}\right)$,

$$
\begin{gathered}
\frac{1}{\mu B^{c l}\left(x_{n}, r_{n}\right)} \int h \mathbf{1}_{B^{c l}\left(x_{n}, r_{n}\right)} d \mu<\frac{2}{\mu B^{c l}\left(x_{n},\left(1+t_{n}\right) r_{n}\right)} \int h \mathbf{1}_{B^{c l}\left(x_{n}, r_{n}\right)} d \mu \\
\leq \frac{2}{\mu B^{c l}\left(y,\left(1+d\left(y, x_{n}\right)\right) r_{n}\right)} \int h \mathbf{1}_{B^{c l}\left(y,\left(1+d\left(y, x_{n}\right)\right) r_{n}\right)} d \mu \leq 2 M h(y) .
\end{gathered}
$$

Therefore

$$
\begin{aligned}
& \int\left(\sum_{1 \leq n \leq N} \frac{w_{n} \mathbf{1}_{B^{c l}\left(x_{n}, r_{n}\right)}}{\mu B^{c l}\left(x_{n}, r_{n}\right)}\right) h d \mu=\sum_{1 \leq n \leq N} \frac{w_{n}}{\mu B^{c l}\left(x_{n}, r_{n}\right)} \int h \mathbf{1}_{B^{c l}\left(x_{n}, r_{n}\right)} d \mu \\
\leq & 2 \sum_{1 \leq n \leq N} w_{n} \inf _{x \in B^{c l}\left(x_{n}, t_{n} r_{n}\right)} M h(x) \leq 2 \sum_{1 \leq n \leq N} \frac{w_{n}}{\mu B^{c l}\left(x_{n}, t_{n} r_{n}\right)} \int(M h) \mathbf{1}_{B^{c l}\left(x_{n}, t_{n} r_{n}\right)} d \mu \\
= & 2 \int\left(\sum_{1 \leq n \leq N} \frac{w_{n} \mathbf{1}_{B^{c l}\left(x_{n}, t_{n} r_{n}\right)}}{\mu B^{c l}\left(x_{n}, t_{n} r_{n}\right)}\right) M h d \mu \leq 2\left\|\sum_{1 \leq n \leq N} \frac{w_{n} \mathbf{1}_{B^{c l}\left(x_{n}, t_{n} r_{n}\right)} \| B^{c l}\left(x_{n}, t_{n} r_{n}\right)}{\mu}\right\| M_{\mu} h \|_{q} .
\end{aligned}
$$

Remark 3.13. Regarding Theorem 3.12 in the special case of Lebesgue measure $\lambda^{d}$ in $\mathbb{R}^{d}$, with different distances (and hence different balls) defined by norms, it is known for some balls if $p>1$, and for all balls if $p>3 / 2$, that $\left\|M_{\mu}\right\|_{L^{p}(\mu) \rightarrow L^{p}(\mu)}$ can be given bounds that are independent of the dimension, see [DeGuMa and the references contained therein.

For instance, if $p=2$, by [DeGuMa, Theorem 5.2], we have $\left\|M_{\mu}\right\|_{L^{2}(\mu) \rightarrow L^{2}(\mu)}<140$, where the constant 140 is independent of $d$, and of the norm considered. Thus, (16) becomes

$$
\left\|\sum_{1 \leq n \leq N} \frac{w_{n} \mathbf{1}_{B^{c l}\left(x_{n}, r_{n}\right)}}{\lambda^{d} B^{c l}\left(x_{n}, r_{n}\right)}\right\|_{2} \leq 280\left\|\sum_{1 \leq n \leq N} \frac{w_{n} \mathbf{1}_{B^{c l}\left(x_{n}, t_{n} r_{n}\right)}}{\lambda^{d} B^{c l}\left(x_{n}, t_{n} r_{n}\right)}\right\|_{2},
$$

and if $p \in(1,2)$, then 280 can be replaced by $2(140)^{2 / q}$ by a simple application of Jensen's inequality (cf. [Al1, Theorem 2.10] for more details). Note however that the $T_{n}$ 's must be very small, depending on the dimension $d$. For instance, for large values of $d$ and every $n$, we can take $T_{n}=1 /(2 d)$, since $(1+1 /(2 d))^{d} \approx e^{1 / 2}<2$.

Note also that the constant $2=1+1$ in (16) can be replaced by $1+\varepsilon$, for any fixed $\varepsilon>0$, simply by choosing smaller values of $T_{n}$. 


\section{Applications to maximal operators}

Definition 4.1. Let $1 \leq p \leq \infty$. We say that the metric measure space $(X, d, \mu)$ has the $p$-Boman covering property (or $p$-Boman overlapping property) with constant $C>0$, if for all finite sequences of closed balls $\left\{B^{c l}\left(x_{n}, r_{n}\right)\right\}_{1 \leq n \leq N}$ with $x_{n}$ in the support of $\mu$, of weights $\left\{w_{n}\right\}_{1 \leq n \leq N}, w_{n}>0$, and of dilations $\left\{t_{n}\right\}_{1 \leq n \leq N}, t_{n} \geq 1$,

$$
\left\|\sum_{1 \leq n \leq N} \frac{w_{n} \mathbf{1}_{B^{c l}\left(x_{n}, t_{n} r_{n}\right)}}{\mu B^{c l}\left(x_{n}, t_{n} r_{n}\right)}\right\|_{p} \leq C\left\|\sum_{1 \leq n \leq N} \frac{w_{n} \mathbf{1}_{B^{c l}\left(x_{n}, r_{n}\right)}}{\mu B^{c l}\left(x_{n}, r_{n}\right)}\right\|_{p} .
$$

The generalized $p$-Boman covering property with constant $C>0$, is defined analogously, but with measurable sets of positive measure replacing the smaller balls. More precisely, the generalized p-Boman covering property holds if for all finite sequences of closed balls $\left\{B^{c l}\left(x_{n}, r_{n}\right)\right\}_{1 \leq n \leq N}$ with $x_{n}$ in the support of $\mu$, of weights $\left\{w_{n}\right\}_{1 \leq n \leq N}, w_{n}>0$, and of sets $\left\{E_{n}\right\}_{1 \leq n \leq N}$ with $\mu E_{n}>0$ and $E_{n} \subset B^{c l}\left(x_{n}, r_{n}\right)$, we have

$$
\left\|\sum_{1 \leq n \leq N} \frac{w_{n} \mathbf{1}_{B^{c l}\left(x_{n}, r_{n}\right)}}{\mu B^{c l}\left(x_{n}, r_{n}\right)}\right\|_{p} \leq C\left\|_{1 \leq n \leq N} \frac{w_{n} \mathbf{1}_{E_{n}}}{\mu E_{n}}\right\|_{p} .
$$

Remark 4.2. Observe that the generalized 1-Boman overlapping property always holds, with $C=1$, since

$$
\left\|\sum_{1 \leq n \leq N} \frac{w_{n} \mathbf{1}_{B^{c l}\left(x_{n}, r_{n}\right)}}{\mu B^{c l}\left(x_{n}, r_{n}\right)}\right\|_{1}=\sum_{1 \leq n \leq N} w_{n}=\left\|\sum_{1 \leq n \leq N} \frac{w_{n} \mathbf{1}_{E_{n}}}{\mu E_{n}}\right\|_{1} .
$$

On the other hand, the $\infty$-Boman overlapping property will rarely hold (it holds if $X$ is finite, for instance). It fails for very well behaved spaces, such as the Lebesgue probability $\lambda$ in $[0,1]$, as we show next. For $1 \leq n \leq N$, set $B^{c l}\left(x_{n}, r_{n}\right)=\left[2^{-n}, 2^{-n+1}\right], w_{n}=2^{-n}=$ $\lambda B^{c l}\left(x_{n}, r_{n}\right)$, and $t_{n}=3$. Since $x_{n}=3 \times 2^{-n-1}$ and $r_{n}=2^{-n-1}$,

$$
\left\|\sum_{1 \leq n \leq N} \frac{w_{n} \mathbf{1}_{B^{c l}\left(x_{n}, t_{n} r_{n}\right)}}{\lambda B^{c l}\left(x_{n}, t_{n} r_{n}\right)}\right\|_{\infty}=\frac{N}{3},
$$

while

$$
\left\|\sum_{1 \leq n \leq N} \frac{w_{n} \mathbf{1}_{B^{c l}\left(x_{n}, r_{n}\right)}}{\lambda B^{c l}\left(x_{n}, r_{n}\right)}\right\|_{\infty}=1
$$

Thus, the $p$-Boman overlapping property and its generalized versions are interesting mostly for $1<p<\infty$.

In [HKST, p. 73] the conclusion of the Vitali covering theorem is turned into a definition as follows: 
Definition 4.3. We say that $(X, d, \mu)$ is a Vitali metric measure space if for all $A \subset X$ and every covering $\mathcal{B}$ of $A$ by closed balls satisfying

$$
\sup _{x \in A} \inf \left\{r>0: B^{c l}(x, r) \in \mathcal{B}\right\}=0,
$$

there exists a disjoint subcollection $\mathcal{C} \subset \mathcal{B}$ such that

$$
\mu(A \backslash \cup \mathcal{C})=0 \text {. }
$$

Of course, if $\mu$ is doubling then $X$ is Vitali, but the latter is a much weaker hypothesis. For instance, it holds for volume on a Riemannian manifold (cf. [HKST, Examples p. 76-77] for this and for other examples).

A standard application of Hölder's inequality leads us from the generalized $q$-Boman covering property to the weak type $(p, p)$ of the centered maximal operator $M$, and from the $q$-Boman covering property to the weak type $(p, p)$ of $M$ for Vitali metric measure spaces. As we shall see below, the $q$-Boman covering property is strictly weaker than the strong $(p, p)$ type of $M^{u}$. Thus, proving the $q$-Boman covering property in cases where the boundedness of $M^{u}$ is not known, or it is known to fail, may yield new results for the centered operator.

But while this property ought to be easier to obtain than the strong type of the maximal operator, since it is weaker, in fact it is not obvious how to do so. Hence, we define weaker properties, in the line of the conclusion of Theorem 3.12, which still yield the above results.

Definition 4.4. Let $1 \leq p \leq \infty$. We say that the metric measure space $(X, d, \mu)$ has the weak $p$-Boman covering property with constant $C>0$, if for every $r>0$ there exists a $T=T(r) \in(0,1)$ such that for all finite sequences of closed balls $\left\{B^{c l}\left(x_{n}, r_{n}\right)\right\}_{1 \leq n \leq N}$ with $x_{n}$ in the support of $\mu$, of weights $\left\{w_{n}\right\}_{1 \leq n \leq N}, w_{n}>0$, and of contractions $\left\{t_{n}\right\}_{1 \leq n \leq N}$, $0<t_{n} \leq T\left(r_{n}\right)$, we have

$$
\left\|\sum_{1 \leq n \leq N} \frac{w_{n} \mathbf{1}_{B^{c l}\left(x_{n}, r_{n}\right)}}{\mu B^{c l}\left(x_{n}, r_{n}\right)}\right\|_{p} \leq C\left\|\sum_{1 \leq n \leq N} \frac{w_{n} \mathbf{1}_{B^{c l}\left(x_{n}, t_{n} r_{n}\right)}}{\mu B^{c l}\left(x_{n}, t_{n} r_{n}\right)}\right\|_{p} .
$$

The generalized weak $p$-Boman covering property is defined analogously, with sets of positive measure contained in the smaller balls replacing the latter.

Theorem 4.5. Let $1<p<\infty$ and let $(X, d, \mu)$ be a metric measure space satisfying a generalized weak $q$-Boman covering property with constant $C$. Then the centered maximal operator $M$ is of weak type $(p, p)$, with $\|M\|_{L^{p} \rightarrow L^{p, \infty}} \leq C$.

Proof. Fix $\varepsilon>0$, let $a>0$ be such that $\mu\{M f>a\}>0$, and let $f \in L^{p}(\mu)$. Now let $E:=\{M f>a\} \cap \operatorname{supp} \mu$, where supp $\mu$ denotes the support of $\mu$. Since the complement of the support $(\operatorname{supp} \mu)^{c}:=\cup\{B(x, r): x \in X, \mu B(x, r)=0\}$ has measure zero by $\tau$-additivity, $\mu E=\mu\{M f>a\}$.

For each $x \in E$, select $B^{c l}(x, r)$ such that $a \mu B^{c l}(x, r)<\int_{B^{c l}(x, r)}|f| d \mu$, and choose $T_{x} \in$ $(0, T(r)]$ with $B^{c l}\left(x, T_{x} r\right) \subset\{M f>a\}$. Define $\mathcal{B}:=\left\{B\left(x, t_{x} r\right): x \in E, 0<t_{x}<T_{x}\right\}$. By $\tau$-additivity, there is a finite subcollection $\mathcal{C}=\left\{B\left(x_{i}, t_{i} r_{i}\right): 1 \leq i \leq N\right\}$ such that

$$
(1-\varepsilon) \mu E=(1-\varepsilon) \mu \cup \mathcal{B}<\mu \cup \mathcal{C} \leq \mu \cup_{i=1}^{N} B^{c l}\left(x_{i}, t_{i} r_{i}\right) .
$$


Furthermore, we may assume that for each $1 \leq i \leq N, \mu B^{c l}\left(x_{i}, t_{i} r_{i}\right) \backslash \cup_{j \neq i}^{N} B^{c l}\left(x_{j}, t_{j} r_{j}\right)>0$, for otherwise we simply disregard whichever ball is almost contained in the union of the others, and this does not change the measure of the finite union. Define the disjointifications $E_{1}=B^{c l}\left(x_{1}, t_{1} r_{1}\right)$, and for $j>1, E_{j}=B^{c l}\left(x_{j}, t_{j} r_{j}\right) \backslash \cup_{i=1}^{j-1} B^{c l}\left(x_{i}, t_{i} r_{i}\right)$. It follows from the generalized weak $q$-Boman covering property, with $w_{i}:=\mu E_{i}>0$, that

$$
\begin{gathered}
(1-\varepsilon) \mu\{M f>a\} \leq \mu \cup_{i=1}^{N} B^{c l}\left(x_{i}, t_{i} r_{i}\right)=\sum_{i=1}^{N} \mu E_{i} \\
=\sum_{i=1}^{N} \frac{\mu E_{i}}{\mu B^{c l}\left(x_{i}, r_{i}\right)} \int_{X} \mathbf{1}_{B^{c l}\left(x_{i}, r_{i}\right)} d \mu \leq \sum_{i=1}^{N} \frac{\mu E_{i}}{\mu B^{c l}\left(x_{i}, r_{i}\right)} \frac{1}{a} \int_{X}|f| \mathbf{1}_{B^{c l}\left(x_{i}, r_{i}\right)} d \mu \\
=\frac{1}{a} \int_{X}|f| \sum_{i=1}^{N} \frac{\mu E_{i}}{\mu B^{c l}\left(x_{i}, r_{i}\right)} \mathbf{1}_{B^{c l}\left(x_{i}, r_{i}\right)} d \mu \leq \frac{1}{a}\|f\|_{p}\left\|\sum_{i=1}^{N} \frac{\mu E_{i}}{\mu B^{c l}\left(x_{i}, r_{i}\right)} \mathbf{1}_{B^{c l}\left(x_{i}, r_{i}\right)}\right\|_{q} \\
\leq \frac{C}{a}\|f\|_{p}\left\|\sum_{i=1}^{N} \mathbf{1}_{E_{i}}\right\|_{q} \leq \frac{C}{a}\|f\|_{p} \mu\{M f>a\}^{1 / q} .
\end{gathered}
$$

Dividing both sides of

$$
(1-\varepsilon) \mu\{M f>a\} \leq \frac{C}{a}\|f\|_{p} \mu\{M f>a\}^{1 / q} .
$$

by $\mu\{M f>a\}^{1 / q}$ and letting $\varepsilon \downarrow 0$ we obtain the result.

Next we indicate the changes needed in the proof when only the weak $q$-Boman covering property is assumed, but in exchange $X$ is taken to be Vitali.

Theorem 4.6. Let $1<p<\infty$ and let $(X, d, \mu)$ be a Vitali metric measure space satisfying a weak $q$-Boman covering property with constant $C$. Then the centered maximal operator $M$ is of weak type $(p, p)$, with $\|M\|_{L^{p} \rightarrow L^{p, \infty}} \leq C$.

Proof. With the same notation as in the previous proof, for each $x \in E$ we select $B^{c l}(x, r)$ such that $a \mu B^{c l}(x, r)<\int_{B^{c l}(x, r)}|f| d \mu$, and choose $T_{x} \in(0, T(r)]$ with $B^{c l}\left(x, T_{x} r\right) \subset\{M f>a\}$. Define $\mathcal{B}:=\left\{B^{c l}\left(x, t_{x} r\right): x \in E, 0<t_{x}<T_{x}\right\}$, so $\mathcal{B}$ is a Vitali cover of $E$. Let $\mathcal{C} \subset \mathcal{B}$ be a (finite or countable) disjoint subcollection with $\mu(E \backslash \cup \mathcal{C})=0$, say, $\mathcal{C}=\left\{B^{c l}\left(x_{i}, t_{i} r_{i}\right): i \in I\right\}$, where either $I=\{1, \ldots, n\}$ for some $n \geq 1$, or $I=\mathbb{N} \backslash\{0\}$. By countable additivity there is a finite $N \geq 1$ such that

$$
(1-\varepsilon) \mu E<\mu \cup_{i=1}^{N} B^{c l}\left(x_{i}, t_{i} r_{i}\right)=\sum_{i=1}^{N} \mu B^{c l}\left(x_{i}, t_{i} r_{i}\right),
$$

and now, taking $w_{i}:=\mu B^{c l}\left(x_{i}, t_{i} r_{i}\right)>0$, the rest of the argument is as before.

Remark 4.7. We do not know if the Vitali assumption can be removed from the preceding theorem. It can be relaxed, however, to the assumption that the Lebesgue differentiation theorem holds for the functions in $L^{p}$, by $[\mathrm{Ha}]$. 
After some preparation, we show that there exists a metric measure space where the uncentered maximal operator satisfies no strong bounds for $1<q<\infty$, and still $p$-Boman holds for every $p \in[1, \infty)$.

Definition 4.8. A Borel measure $\mu$ on $(X, d)$ is doubling at large scales, or doubling localized at radii larger than $R$, if there exists an $R>0$ and a $C \geq 1$ such that for all $r>R$ and all $x \in X, \mu(B(x, 2 r)) \leq C \mu(B(x, r))<\infty$.

Definition 4.9. A Borel measure $\mu$ on $(X, d)$ is doubling on a subset $E \subset X$, if there exists a $C \geq 1$ such that for all $x \in E, \mu(B(x, 2 r)) \leq C \mu(B(x, r))<\infty$.

The next example will be used later, in Theorem 4.12.

Example 4.10. There exists a Vitali metric measure space $(X, d, \mu)$ such that for each $1 \leq$ $p<\infty, M^{u}$ is not of weak type $(p, p)$, but $\mu$ it is doubling localized at radii larger than $R>0$, for any such $R$. In fact, if $R \geq 2^{-n}$, we can take $C=2^{3+n}$.

The set $X$ will be a suitable subset of $\mathbb{R}^{2}$ with distance defined by the $\ell_{1}$ norm: Given $x=\left(x_{1}, x_{2}\right)$ and $y=\left(y_{1}, y_{2}\right), d(x, y)=\|x-y\|_{1}=\left|x_{1}-y_{1}\right|+\left|x_{2}-y_{2}\right|$. By the Besicovitch covering theorem, $X$ is Vitali.

Let $A_{n}:=\left[4 n, 4 n+2^{-n}\right] \times\left\{2^{-n}\right\}$, and let $A:=(-\infty, \infty) \times\{0\}$ be the $x$ axis. Set $X:=A \cup\left(\cup_{n \geq 0} A_{n}\right)$, and define $\mu$ restricted to $A$ as linear Lebesgue measure $\lambda$, while $\mu$ restricted to $A_{n}$ is $2^{-n} \lambda$, the linear Lebesgue measure scaled by a factor of $2^{-n}$; thus, $\mu A_{n}=$ $4^{-n}$. Clearly the failure of doubling occurs at small radii: $\mu B_{1}^{c l}\left(\left(4 n, 2^{-n}\right), 2^{-n}\right)=4^{-n}$, while $\mu B_{1}^{c l}\left(\left(4 n, 2^{-n}\right), 2^{-n+1}\right)=4^{-n}+2^{1-n}$.

Lack of doubling also follows from the fact that $M_{\mu}^{u}$ is not of strong type $(p, p)$ for any $1<p<\infty$ (and hence, it is not of weak type $(p, p)$ either). Since $\left\|\mathbf{1}_{A_{n}}\right\|_{p}^{p}=4^{-n}$, and $M_{\mu}^{u} \mathbf{1}_{A_{n}} \equiv 1$ on $\left[4 n, 4 n+2^{-n}\right] \times\{0\}$, we have $\left\|M_{\mu}^{u} \mathbf{1}_{A_{n}}\right\|_{p}^{p}>2^{n}\left\|\mathbf{1}_{A_{n}}\right\|_{p}^{p}$.

We prove by induction on $n$, where $R \geq 2^{-n}$, that $\mu$ is doubling in the large. For every $x \in A$,

$$
\begin{aligned}
& \mu B(x, 2 r)=\mu(A \cap B(x, 2 r))+\mu\left(\cup_{n \geq 0} A_{n} \cap B(x, 2 r)\right) \\
& \leq 2 \mu(A \cap B(x, 2 r))=4 \mu(A \cap B(x, r)) \leq 4 \mu(B(x, r)),
\end{aligned}
$$

so on $A, \mu$ is actually doubling, and we only need to consider balls with centers in $\cup_{n \geq 0} A_{n}$; this is always assumed from now on. Denote by $\pi_{1}(a, b):=(a, 0)$ the projection onto the first coordinate, and observe that for every $x \in \cup_{n \geq 0} A_{n}$,

$$
\mu B(x, 2 r) \leq \mu\left(B\left(\pi_{1} x, 2 r\right)\right) \leq 8 r .
$$

Now the claim to be shown is that if $R \geq 2^{-n}$, then $C=C(R) \geq \mu B(x, 2 r) / \mu(B(x, r))$ can be taken to be equal to $2^{3+n}$. Note that if $R \geq 2^{-n}$, then there is no loss of generality in assuming that $R=2^{-n}$, since more radii are being considered in this case.

Let $n=0$, so $r>R=1$. If $x \in A_{N}, N>0$, then

$$
\mu B(x, r) \geq \mu(A \cap B(x, r)) \geq 2 r-1 \geq r
$$


hence, we can take $C=2^{3+0}$.

And if $x \in A_{0}$, for $r \geq 2$,

$$
\mu B(x, r) \geq \mu(A \cap B(x, r)) \geq 2 r-2 \geq r
$$

while if $1<r<2$, then

$$
\mu B(x, 2 r) \leq 1+4 r-2<4 r
$$

and $\mu B(x, r) \geq 1>r / 2$, so once again we can take $C=2^{3+0}$.

The general case $R \geq 2^{-n}$ is obtained in a similar fashion. If $r>2^{-n+1}$ we take $R=2^{-n+1}$ and apply the induction hypothesis, which we assume for $n-1 \geq 0$, and prove for $n$. As before, we may suppose that $R=2^{-n}$ and $2^{-n+1} \geq r>2^{-n}$. Let $x \in A_{N}$. The restriction of $\mu$ to $A_{N}$ is doubling with constant 2 , so if $N<n-1$, then $\mu B(x, 2 r) \leq 2 \mu(B(x, r))$. If $N=n-1$, then $\mu B(x, r) \geq 2^{-n+1} r$, if $N=n$, then $\mu B(x, r) \geq 2^{-n} r$, and if $N>n$, then

$$
\mu B(x, r) \geq \mu(A \cap B(x, r)) \geq 2 r-2^{-n}>r,
$$

so it follows from (25) that in every case $\mu B(x, 2 r) / \mu(B(x, r)) \leq 2^{3+n}$.

The standard argument from doubling to weak type $(1,1)$ yields the corresponding results when we localize at large scales, and when we work with measures doubling on subsets.

Theorem 4.11. Let $(X, d, \mu)$ be a metric measure space. If $\mu$ is doubling at large scales, say, localized at radii larger than $R>0$, with doubling constant $C=C(R)$, then the uncentered localized maximal operator $M_{\{R<r\}, \mu}^{u}$ is of weak type $(1,1)$ with constant $C^{3}$, and thus, of strong type $(p, p)$ for all $p>1$, with constant $C=\frac{C^{3 / p} p}{p-1}$.

Likewise, if $\mu$ is doubling on $E \subset X$, with constant $C$, then for every $t>0$ and every $g \in L^{1}(\mu)$,

$$
\mu\left(E \cap\left\{M_{\mu}^{u} g>t\right\}\right) \leq \frac{C^{3}\|g\|_{L^{1}(\mu)}}{t} .
$$

Thus, if $f \in L^{p}(\mu)$ for some $p>1$,

$$
\left\|\mathbf{1}_{E} M_{\mu}^{u} f\right\|_{p} \leq \frac{C^{3 / p} p}{p-1}\|f\|_{p}
$$

In metric spaces neither the centers nor the radii of balls are in general unique. We use the standard convention whereby when $B$ is used as an abbreviation of $B(x, r)$, we are implicitly keeping the same $x$ and the same $r$, and when $t>0, t B$ is understood as $B(x, t r)$.

Theorem 4.12. There exists a Vitali metric measure space $(X, d, \mu)$ satisfying a p-Boman covering property for each $1<p<\infty$, such that for all $q \in(1, \infty),\left\|M_{\mu}^{u}\right\|_{L^{q}(\mu) \rightarrow L^{q}(\mu)}=\infty$.

Proof. We use the Vitali metric measure space $(X, d, \mu)$ defined in example 4.10, It was already noted there that $M_{\mu}^{u}$ is not of strong type $(q, q)$ for any $1<q<\infty$. Fix $p \in(1, \infty)$.

To check that for some $C>0$ (whose value will be determined later)

$$
\left\|\sum_{1 \leq n \leq N} \frac{w_{n} \mathbf{1}_{B^{c l}\left(x_{n}, t_{n} r_{n}\right)}}{\mu B^{c l}\left(x_{n}, t_{n} r_{n}\right)}\right\|_{p} \leq C\left\|\sum_{1 \leq n \leq N} \frac{w_{n} \mathbf{1}_{B^{c l}\left(x_{n}, r_{n}\right)}}{\mu B^{c l}\left(x_{n}, r_{n}\right)}\right\|_{p},
$$


we split the sum in the left hand side into smaller sums, and then we use the triangle inequality. Without loss of generality, we may assume that the balls $\left\{B^{c l}\left(x_{n}, t_{n} r_{n}\right): 1 \leq n \leq N\right\}$ are ordered by decreasing radii $t_{n} r_{n}$. Let $k_{1}$ be such that for $1 \leq n \leq k_{1}, t_{n} r_{n}>1$, and for $k_{1}<n \leq N, t_{n} r_{n} \leq 1$. Note that if $k_{1}<1$ (that is, $t_{n} r_{n} \leq 1$ always) then the first sum is empty and we can go directly to the second step, while if $k_{1}=N$ (so $t_{n} r_{n}>1$ for every $n$ ) then the first sum is the whole sum and the proof finishes in one step. So suppose $1 \leq k_{1}<N$. Now by Example 4.10 and Theorem 4.11, the uncentered localized maximal operator $M_{\left\{2^{0}<r\right\}, \mu}^{u}$ is of strong type $(q, q)$, with constant $C=\frac{8^{3 / q} q}{q-1}$. Thus

$$
I:=\left\|\sum_{1 \leq n \leq k_{1}} \frac{w_{n} \mathbf{1}_{B^{c l}\left(x_{n}, t_{n} r_{n}\right)}}{\mu B^{c l}\left(x_{n}, t_{n} r_{n}\right)}\right\|_{p} \leq C_{1}\left\|\sum_{1 \leq n \leq N} \frac{w_{n} \mathbf{1}_{B^{c l}\left(x_{n}, r_{n}\right)}}{\mu B^{c l}\left(x_{n}, r_{n}\right)}\right\|_{p},
$$

with $C_{1}=\frac{8^{3 / q} q}{q-1}$.

Recall that $X=A \cup\left(\cup_{n \geq 0} A_{n}\right)$, where $A_{n}:=\left[4 n, 4 n+2^{-n}\right] \times\left\{2^{-n}\right\}$ and $A:=(-\infty, \infty) \times\{0\}$, and the distance on $X$ is defined by restriction of the $\ell_{1}$ norm on the plane. By reordering the balls $\left\{B^{c l}\left(x_{n}, t_{n} r_{n}\right): k_{1}<n \leq N\right\}$ in such a way that the ones centered at $A$ appear first in the list, we may suppose that there exists a $k_{2} \in\left(k_{1}, N\right]$ such that if $k_{1}<n \leq k_{2}$, then $x_{n} \in A$, while if $n>k_{2}$, then $x \in \cup_{n \geq 0} A_{n}$. Of course, if $k_{2}=N$ then the set of indices $n$ satisfying $n>k_{2}$ is empty, and the proof finishes with the next step. Set

$$
I I:=\left\|\sum_{k_{1}<n \leq k_{2}} \frac{w_{n} \mathbf{1}_{B^{c l}\left(x_{n}, t_{n} r_{n}\right)}}{\mu B^{c l}\left(x_{n}, t_{n} r_{n}\right)}\right\|_{p} .
$$

Note that for every $y \in X$,

$$
\sum_{k_{1}<n \leq k_{2}} \frac{w_{n} \mathbf{1}_{B^{c l}\left(x_{n}, t_{n} r_{n}\right)}}{\mu B^{c l}\left(x_{n}, t_{n} r_{n}\right)}(y) \leq \sum_{k_{1}<n \leq k_{2}} \frac{w_{n} \mathbf{1}_{B^{c l}\left(x_{n}, t_{n} r_{n}\right)}}{\mu B^{c l}\left(x_{n}, t_{n} r_{n}\right)}\left(\pi_{1}(y)\right),
$$

SO

$$
\begin{aligned}
\sum_{k_{1}<n \leq k_{2}} \frac{w_{n} \mathbf{1}_{B^{c l}\left(x_{n}, t_{n} r_{n}\right)}}{\mu B^{c l}\left(x_{n}, t_{n} r_{n}\right)}= & \mathbf{1}_{A} \sum_{k_{1}<n \leq k_{2}} \frac{w_{n} \mathbf{1}_{B^{c l}\left(x_{n}, t_{n} r_{n}\right)}}{\mu B^{c l}\left(x_{n}, t_{n} r_{n}\right)}+\mathbf{1}_{\cup_{n \geq 0} A_{n}} \sum_{k_{1}<n \leq k 2} \frac{w_{n} \mathbf{1}_{B^{c l}\left(x_{n}, t_{n} r_{n}\right)}}{\mu B^{c l}\left(x_{n}, t_{n} r_{n}\right)} \\
& \leq 2 \times \mathbf{1}_{A} \sum_{k_{1}<n \leq k_{2}} \frac{w_{n} \mathbf{1}_{B^{c l}\left(x_{n}, t_{n} r_{n}\right)}}{\mu B^{c l}\left(x_{n}, t_{n} r_{n}\right)}
\end{aligned}
$$

It follows that

$$
I I \leq 2\left\|\mathbf{1}_{A} \sum_{k_{1}<n \leq k_{2}} \frac{w_{n} \mathbf{1}_{B^{c l}\left(x_{n}, t_{n} r_{n}\right)}}{\mu B^{c l}\left(x_{n}, t_{n} r_{n}\right)}\right\|_{p} .
$$

Now for every measurable set $E \subset X, \mu E \leq 2 \mu\left(\pi_{1} E\right)$, so $\mu$ is doubling on $A$, with doubling constant $C \leq 8$ (the bound from (25), which is not tight on $A$ ) so by Theorem 4.11 once 
more,

$$
I I \leq 2 \frac{8^{3 / q} q}{q-1}\left\|\sum_{1 \leq n \leq N} \frac{w_{n} \mathbf{1}_{B^{c l}\left(x_{n}, r_{n}\right)}}{\mu B^{c l}\left(x_{n}, r_{n}\right)}\right\|_{p}
$$

and thus, we can take $C_{2}=2 C_{1}$.

Finally, if the set of indices $n$ satisfying $n>k_{2}$ is not empty, for each such $n$ the ball $B^{c l}\left(x_{n}, t_{n} r_{n}\right)$ has radius bounded by 1 and center contained in some $A_{N}, N \geq 1$. It follows that if $j>k_{2}$ and $B^{c l}\left(x_{n}, t_{n} r_{n}\right) \cap B^{c l}\left(x_{j}, t_{j} r_{j}\right) \neq \emptyset$, then $x_{j} \in A_{N}$ also. We need to bound

$$
I I I:=\left\|\sum_{k_{2}<n \leq N} \frac{w_{n} \mathbf{1}_{B^{c l}\left(x_{n}, t_{n} r_{n}\right)}}{\mu B^{c l}\left(x_{n}, t_{n} r_{n}\right)}\right\|_{p} \text {. }
$$

Let $\left\{A_{N_{1}}, \ldots, A_{N_{s}}\right\}$ be precisely the set of $A_{n}$ 's that intersect (or equivalently, contain the center) of at least one of the balls $B^{c l}\left(x_{n}, t_{n} r_{n}\right), n>k_{2}$. For each $1 \leq j \leq s$, let $\mathcal{C}_{j}:=$ $\left\{B^{c l}\left(x_{j_{1}}, t_{j_{1}} r_{j_{1}}\right), \ldots, B^{c l}\left(x_{j_{s(j)}}, t_{j_{s(j)}} r_{j_{s(j)}}\right)\right\}$ be the collection of all balls with radius bounded by 1 and with center in $A_{N_{j}}$. Set

$$
G_{j}(x):=\sum_{1 \leq i \leq s(j)} \frac{w_{n} \mathbf{1}_{B^{c l}\left(x_{j_{i}}, t_{j_{i}} r_{j_{i}}\right)}}{\mu B^{c l}\left(x_{j_{i}}, t_{j_{i}} r_{j_{i}}\right)}(x) \text { and } g_{j}(x):=\sum_{1 \leq i \leq s(j)} \frac{w_{n} \mathbf{1}_{B^{c l}\left(x_{j_{i}}, r_{j_{i}}\right)}}{\mu B^{c l}\left(x_{j_{i}}, r_{j_{i}}\right)}(x) .
$$

Since for $i \neq j$ the functions $G_{i}$ and $G_{j}$ have disjoint supports, it is enough to show that for a suitable constant $C_{3}$, we have $I I I_{j}^{p}:=\int G_{j}^{p} d \mu \leq C_{3}^{p} \int g_{j}^{p} d \mu$.

Again we break up each of these sums $G_{j}$ into different smaller sums (not necessarily disjoint, so some balls may appear more than once).

First of all, consider the collection $\mathcal{C}_{j, 1}$ of balls $B^{c l}\left(x_{j_{i}}, t_{j_{i}} r_{j_{i}}\right)$ in $\mathcal{C}_{j}$ such that

$$
\mu\left(B^{c l}\left(x_{j_{i}}, t_{j_{i}} r_{j_{i}}\right) \cap A\right) \leq \mu\left(B^{c l}\left(x_{j_{i}}, t_{j_{i}} r_{j_{i}}\right) \cap A_{N_{j}}\right) .
$$

To avoid more subscripts, we rename the balls in $\mathcal{C}_{j, 1}$ as $U_{1}, \ldots, U_{m}$, and we call $u_{1}, \ldots, u_{m}$ the corresponding contracted balls. Note then that $\mu\left(u_{j} \cap A\right) \leq \mu\left(u_{j} \cap A_{N_{j}}\right)$, since either $u_{j} \cap A=\emptyset$, or if $u_{j} \cap A \neq \emptyset$, then $u_{j} \cap A_{N_{j}}=A_{N_{j}}$, so

$$
\mu\left(u_{j} \cap A\right) \leq \mu\left(U_{j} \cap A\right) \leq \mu\left(U_{j} \cap A_{N_{j}}\right)=\mu\left(A_{N_{j}}\right)=\mu\left(u_{j} \cap A_{N_{j}}\right) .
$$

Since $\mu$ on $A_{N_{j}}$ is just a multiple of the one dimensional Lebesgue measure $\lambda$, we have

$$
\left\|M_{\mu}^{u}\right\|_{L^{q}\left(A_{N_{j}}\right) \rightarrow L^{q}\left(A_{N_{j}}\right)} \leq \frac{2^{1 / q} q}{q-1}
$$

for, as is well known, $\left\|M_{\lambda}^{u}\right\|_{L^{1}\left(A_{N_{j}}\right) \rightarrow L^{1, \infty}\left(A_{N_{j}}\right)} \leq 2$.

Thus,

$$
\left\|\sum_{1 \leq n \leq m} \frac{w_{n} \mathbf{1}_{U_{n}}}{\mu U_{n}}\right\|_{p} \leq\left\|\sum_{1 \leq n \leq m} \frac{2 w_{n} \mathbf{1}_{\left(U_{n} \cap A_{N_{j}}\right)}}{\mu\left(U_{n} \cap A_{N_{j}}\right)}\right\|_{p} \leq \frac{2^{1 / q} q}{q-1}\left\|_{1 \leq n \leq m} \frac{2 w_{n} \mathbf{1}_{\left(u_{n} \cap A_{N_{j}}\right)}}{\mu\left(u_{n} \cap A_{N_{j}}\right)}\right\|_{p}
$$




$$
\leq \frac{2^{1 / q} q}{q-1}\left\|\sum_{1 \leq n \leq m} \frac{4 w_{n} \mathbf{1}_{u_{n}}}{\mu u_{n}}\right\|_{p}=\frac{2^{1 / q} 4 q}{q-1}\left\|\sum_{1 \leq n \leq m} \frac{w_{n} \mathbf{1}_{u_{n}}}{\mu u_{n}}\right\|_{p} .
$$

Let $C_{4}=\frac{2^{1 / q} 4 q}{q-1}$. Next, consider the collection $\mathcal{C}_{j, 2}$ of balls $B^{c l}\left(x_{j_{i}}, t_{j_{i}} r_{j_{i}}\right)$ such that

$$
\mu\left(B^{c l}\left(x_{j_{i}}, t_{j_{i}} r_{j_{i}}\right) \cap A\right) \geq \mu\left(B^{c l}\left(x_{j_{i}}, t_{j_{i}} r_{j_{i}}\right) \cap A_{N_{j}}\right) .
$$

We rename the balls in $\mathcal{C}_{j, 2}$, ordering them by decreasing radii, as $V_{1}, \ldots, V_{\ell}$, and we call $v_{1}, \ldots, v_{\ell}$ the corresponding contracted balls. Now let $k$ be such that for $1 \leq i \leq k, \mu\left(v_{i} \cap A\right) \geq$ $\mu\left(v_{i} \cap A_{N_{j}}\right)$, while for $k<i \leq \ell, \mu\left(v_{i} \cap A\right)<\mu\left(v_{i} \cap A_{N_{j}}\right)$. Of course, it might happen that $k<1$ (when $\mu\left(v_{i} \cap A\right)<\mu\left(v_{i} \cap A_{N_{j}}\right)$ for all balls) or that $k=\ell\left(\right.$ when $\mu\left(v_{i} \cap A\right) \geq \mu\left(v_{i} \cap A_{N_{j}}\right)$ for every $1 \leq i \leq \ell)$. In either case, we just have one fewer sum to consider.

Now, regarding the balls $V_{1}, \ldots, V_{k}$, the situation is essentially the same as when bounding $I I$ :

$$
\begin{gathered}
\left\|\sum_{1 \leq n \leq k} \frac{w_{n} \mathbf{1}_{V_{n}}}{\mu V_{n}}\right\|_{p} \leq\left\|\sum_{1 \leq n \leq k} \frac{2 w_{n} \mathbf{1}_{\left(V_{n} \cap A\right)}}{\mu\left(V_{n} \cap A\right)}\right\|_{p} \leq C_{2}\left\|\sum_{1 \leq n \leq k} \frac{2 w_{n} \mathbf{1}_{\left(v_{n} \cap A\right)}}{\mu\left(v_{n} \cap A\right)}\right\|_{p} \\
\leq C_{2}\left\|\sum_{1 \leq n \leq k} \frac{4 w_{n} \mathbf{1}_{v_{n}}}{\mu v_{n}}\right\|_{p}=4 C_{2}\left\|\sum_{1 \leq n \leq k} \frac{w_{n} \mathbf{1}_{v_{n}}}{\mu v_{n}}\right\|_{p} .
\end{gathered}
$$

As for the balls $V_{k+1}, \ldots, V_{\ell}$, for each $i \in(k, \ell]$ choose $t_{i} \in(0,1]$ so that $\mu\left(t_{i} V_{i} \cap A\right)=$ $\mu\left(t_{i} V_{i} \cap A_{N_{j}}\right)$. This is always possible to do, since $\mu$ is a continuous measure, and whenever $t_{i} V_{i} \cap A \neq \emptyset$, we have $t_{i} V_{i} \cap A_{N_{j}}=V_{i} \cap A_{N_{j}}=A_{N_{j}}$. But now the problem has been reduced to the preceding two cases, successively considered:

$$
\left\|\sum_{1 \leq n \leq k} \frac{w_{n} \mathbf{1}_{V_{n}}}{\mu V_{n}}\right\|_{p} \leq 4 C_{2}\left\|\sum_{1 \leq n \leq k} \frac{w_{n} \mathbf{1}_{t_{n} V_{n}}}{\mu t_{n} V_{n}}\right\|_{p} \leq 4 C_{2} C_{4}\left\|\sum_{1 \leq n \leq k} \frac{w_{n} \mathbf{1}_{v_{n}}}{\mu v_{n}}\right\|_{p} .
$$

Writing $C_{3}:=C_{4}+4 C_{2}+4 C_{2} C_{4}$ and using the fact that the $G_{i}$ 's are disjointly supported functions (hence, so are the $g_{i}$ 's) we have

$$
\begin{aligned}
& \int\left(\sum_{j=1}^{s} G_{j}\right)^{p} d \mu=\sum_{j=1}^{s} \int G_{j}^{p} d \mu \leq \sum_{j=1}^{s} C_{3}^{p} \int g_{j}^{p} d \mu \\
= & C_{3}^{p} \int\left(\sum_{j=1}^{s} g_{j}\right)^{p} d \mu \leq C_{3}^{p}\left\|\sum_{1 \leq n \leq N} \frac{w_{n} \mathbf{1}_{B^{c l}\left(x_{n}, r_{n}\right)}}{\mu B^{c l}\left(x_{n}, r_{n}\right)}\right\|_{p}^{p} .
\end{aligned}
$$

Putting together the estimates for $I, I I$, and $I I I$, we obtain (26) with $C=C_{1}+C_{2}+C_{3}$.

Remark 4.13. Note that if the $p$-Boman covering property holds for some $p>1$, with constant $C_{p}$, then it holds for all the intermediate spaces $L^{s}(\mu), 1 \leq s \leq p$, with constant bounded by $C_{p}^{\frac{(s-1) p}{s(p-1)}}$. This follows from Riesz-Thorin, applied to the operators defined by 
$T G=g$ on the cone of nonnegative functions of the form $G=\sum_{1 \leq n \leq N} \frac{w_{n} \mathbf{1}_{B^{c l}\left(x_{n}, r_{n}\right)}}{\mu B^{c l}\left(x_{n}, r_{n}\right)}$, with $g=\sum_{1 \leq n \leq N} \frac{w_{n} \mathbf{1}_{B^{c l}\left(x_{n}, t_{n} r_{n}\right)}}{\mu B^{c l}\left(x_{n}, t_{n} r_{n}\right)}$ a contracted version of $G$ (so $0<t_{i} \leq 1,0<w_{i}$ ).

\section{REFERENCES}

[Al1] Aldaz, J. M. Local comparability of measures, averaging and maximal averaging operators. Potential Anal. 49 (2018), no. 2, 309-330. Available at the Mathematics ArXiv.

[A12] Aldaz, J. M. The Stein-Strömberg covering theorem in metric spaces. J. Math. Anal. Appl. 449 (2017), no. 2, 1741-1753.

[A13] Aldaz, J. M. On the pointwise domination of a function by its maximal function. To appear, Arch. Math., available at the Mathematics ArXiv.

[Boj] Bojarski, B. Remarks on Sobolev imbedding inequalities. Complex analysis, Joensuu 1987, 52-68, Lecture Notes in Math., 1351, Springer, Berlin, 1988.

[Bom] Boman, J. L L $^{p}$ estimates for very strongly elliptic systems, Report no. 29, Department of Mathematics, University of Stockholm, Sweden (1982).

[Bu] Buckley, Stephen M. Strong doubling conditions. Math. Inequal. Appl. 1 (1998), no. 4, 533-542.

[Ch] Chua, Seng-Kee Weighted Sobolev inequalities on domains satisfying the chain condition. Proc. Amer. Math. Soc. 117 (1993), no. 2, 449-457.

[Ch1] Chua, Seng-Kee Sobolev interpolation inequalities on generalized John domains. Pacific J. Math. 242 (2009), no. 2, 215-258.

[ChWh] Chua, Seng-Kee; Wheeden, Richard L. Self-improving properties of inequalities of Poincar type on measure spaces and applications. J. Funct. Anal. 255 (2008), no. 11, 2977-3007.

[DeGuMa] Deleaval, L., Guédon, O. and Maurey, B. Dimension free bounds for the Hardy-Littlewood maximal operator associated to convex sets. To appear, Ann. Fac. Sci. Toulouse Math., available at the Math. ArXiv.

[Gra] L. Grafakos, Classical Fourier analysis. Third edition. Graduate Texts in Mathematics, 249. Springer, New York, (2014).

[Ha] Hayes, C. A. Derivation of the integrals of $L^{(q)}$-functions. Pacific J. Math. 64 (1976), 173-180.

[HeKo] Heinonen, Juha; Koskela, Pekka Definitions of quasiconformality. Invent. Math. 120 (1995), no. 1, $61-79$.

[HKST] J. Heinonen, P. Koskela, N. Shanmugalingam, J. T Tyson, Sobolev spaces on metric measure spaces. An approach based on upper gradients. New Mathematical Monographs, 27. Cambridge University Press, Cambridge, 2015.

[Hy] Hytönen, Tuomas A framework for non-homogeneous analysis on metric spaces, and the RBMO space of Tolsa. Publ. Mat. 54 (2010), no. 2, 485-504.

[IhVa] Ihnatsyeva, Lizaveta; Vähäkangas, Antti V. Characterization of traces of smooth functions on Ahlfors regular sets. J. Funct. Anal. 265 (2013), no. 9, 1870-1915.

[Io] Ionescu, Alexandru D. A maximal operator and a covering lemma on non-compact symmetric spaces. Math. Res. Lett. 7 (2000), 83-93.

[IwNo] Iwaniec, T.; Nolder, C. A. Hardy-Littlewood inequality for quasiregular mappings in certain domains in $\mathbb{R}^{n}$. Ann. Acad. Sci. Fenn. Ser. A I Math. 10 (1985), 267-282.

[KoShSt] Kozlovski, O.; Shen, W.; van Strien, S. Rigidity for real polynomials. Ann. of Math. (2) 165 (2007), no. 3, 749-841.

[Str] Strömberg, Jan-Olov Weak type $L^{1}$ estimates for maximal functions on noncompact symmetric spaces. Ann. of Math. (2) 114 (1981), no. 1, 115-126. 
Instituto de Ciencias Matemáticas (CSIC-UAM-UC3M-UCM) and Departamento de Matemáticas, Universidad Autónoma de Madrid, Cantoblanco 28049, Madrid, Spain.

E-mail address: jesus.munarriz@uam.es

E-mail address: jesus.munarriz@icmat.es 